arXiv:0805.0781

\title{
Limits on Neutron Lorentz Violation from the Stability of Primary Cosmic Ray Protons
}

\author{
Brett Altschul] \\ Department of Physics and Astronomy \\ University of South Carolina \\ Columbia, SC 29208 USA
}

\begin{abstract}
Recent evidence appears to confirm that the ultra-high-energy primary cosmic ray spectrum consists mostly of protons. The fact that these protons can traverse large distances to reach Earth allows us to place bounds on Lorentz violations. The protons neither emit vacuum Cerenkov radiation nor $\beta$-decay into neutrons, and this constrains six previously unmeasured coefficients in the neutron sector at the $5 \times 10^{-14}$ level. Among the coefficients bounded here for the first time are those that control spin-independent boost anisotropy for neutrons. This is a phenomenon which could have existed (in light of the preexisting bounds) without additional fine tuning. There are also similar bounds for others species of hadrons. The bounds on Lorentz violation for neutral pions are particularly strong, at the $4 \times 10^{-21}$ level, eleven orders of magnitude better than previous constraints.
\end{abstract}

\footnotetext{
${ }^{1}$ baltschu@physics.sc.edu
} 
The observation [1] of the Greisen-Zatsepin-Kuz'min (GZK) cutoff [2, 3] and the discovery that ultra-high-energy cosmic rays are associated with nearby active galactic nuclei [4] resolved a major puzzle in physics. A number of exotic physical scenarios had been suggested to explain the apparent absence of the GZK cutoff in earlier observations. Such exotic physics would now seem to be unnecessary; however, it is interesting to turn things around and ask what constraints can be placed on exotic theories based on our improved understanding of cosmic ray physics.

One exotic idea which has attracted significant interest in the last decade (and which was put forward to explain the apparent anomalies in the cosmic ray spectrum [5]) is Lorentz violation. There has already been some discussion of how new ultra-high-energy cosmic ray data can be used to constrain Lorentz violation [6, 7, 8, However, there is a great deal more useful information that can still be extracted from what we now know about the highest-energy cosmic rays.

Lorentz violation can be described by an effective field theory, the standard model extension (SME), which contains all possible local Lorentz-violating operators constructed from standard model fields [9, 10]. The minimal SME, which is the standard theory used to parameterize Lorentz tests, is a version of the SME containing only gauge invariant and power-counting renormalizable operators. Many of the minimal SME coupling constants have been constrained extremely tightly, but others have not. (For details, see [11]. Up-todate information on minimal SME bounds can be found in [12.) Here we shall show that cosmic ray observations allow us to place constraints on several previously unbounded parameters. Among these are six SME coefficients in the neutron sector, including three which parameterize spin-independent boost invariance violations (a phenomenon which has never before been studied in this sector).

It may seem surprising that bounds on any neutron coefficients are possible, since neutron physics ordinarily have little to do with primary cosmic rays. However, Lorentz violation could allow otherwise forbidden proton-to-neutron transitions. We therefore wish to emphasize the rather interesting observation that measurements that are entirely concerned with one species of particles (here, protons) can be used to place strong constraints on exotic physics involving different particles (neutrons or other species).

The experimental confirmation that the GZK cutoff does indeed exist at $\sim 6 \times 10^{10}$ $\mathrm{GeV}$ is good evidence that ultra-high-energy primary cosmic rays are mostly protons. That protons should dominate the spectrum was expected originally, but was called into question by the observation of a few cosmic ray events well above the cutoff. However, we can now feel fairly confident (although not absolutely certain) that we understand the protonic cosmic ray spectrum up to the highest energies.

We shall assume that most of the highest energy primary cosmic rays are indeed protons. If this somehow turns out not to be the case, the constraints derived here would be invalidated. Obviously then, further confirmation of the protonic nature of the rays would be helpful.

We will also encounter another caveat that constrains the applicability of our bounds. 
Cosmic ray data imply that the Lorentz violation coefficients from the minimal SME must obey a large number of one-sided inequalities. In order to translate these into two-sided bounds on the individual coefficients, an assumption must be made about the form of the Lorentz violation. This assumption is that isotropic Lorentz violation (pure boost noninvariance) is sufficiently small. This assumption is not presently justified by experiment, but it will nonetheless be made in much of our analysis. Making the assumption allows us to see much more easily which forms of Lorentz violation cosmic ray measurements are sensitive to. This will be discussed in more detail below.

The minimal SME Lagrange density for each spin- $\frac{1}{2}$ fermion species includes terms of the form

$$
\mathcal{L}=\bar{\psi}\left[i\left(\gamma^{\mu}+c^{\nu \mu} \gamma_{\nu}-d^{\nu \mu} \gamma_{\nu} \gamma_{5}\right) \partial_{\mu}-m\right] \psi
$$

There is a different set of $c$ and $d$ coefficients for each species. There are many additional terms in the most general minimal $\mathrm{SME} \mathcal{L}$, but $c$ and $d$ are the most relevant at high energies. The effects of other terms are suppressed in relative importance by powers of $m / E$. There are also analogous coefficients for scalar and vector particles. Since Lorentz violation is known to be a small effect, all the calculations here will be done to leading order in the violation coefficients.

When Lorentz symmetry is broken by $c$ or $d$, novel effects may appear. For example, it may become possible for sufficiently energetic protons to decay. Obviously, this is a unique signature of Lorentz violation. In a Lorentz-symmetric theory, if the decay is forbidden when the proton is at rest, it is forbidden for all proton momenta; and even if the proton is not absolutely stable at rest, Lorentz invariance dictates that it will survive longer when it is highly boosted, because of the time dilation effect.

Of interest are decays of the proton into two or more particles, $p^{+} \rightarrow n+\pi^{+}$, for example. At high energies, the Lorentz violation changes the energy-momentum relation for each particle involved to

$$
E_{w}(\vec{p})=\sqrt{m_{w}^{2}+\left[1+2 \delta_{w}(\hat{p})\right] \vec{p}^{2}}
$$

where $w$ labels the species, and the maximum achievable velocity (MAV) for a species in the direction $\hat{p}$ is $1+\delta_{w}(\hat{p})$. Other forms of Lorentz violation may modify the energymomentum relation at lower energies, but any such effects are suppressed in relative importance by powers of $m_{w} / p$. The parameter $\delta_{w}$ can be spin dependent, and for spin- $\frac{1}{2}$ fermions, it is equal to 13

$$
\delta_{w}(\hat{p})=-c_{00}^{w}-c_{(0 j)}^{w} \hat{p}_{j}-c_{j k}^{w} \hat{p}_{j} \hat{p}_{k}+s d_{00}^{w}+s d_{(0 j)}^{w} \hat{p}_{j}+s d_{j k}^{w} \hat{p}_{j} \hat{p}_{k},
$$

where $s$ is the helicity, $c_{(0 j)}=c_{0 j}+c_{j 0}$ [likewise for $d_{(0 j)}$ ], and the superscripts indicate that the coefficients are those for the species $w$.

Proton decay above a certain threshold could occur if there were a mismatch between the $\delta_{p}$ for the proton and the corresponding parameters for the daughter particles. We shall consider three possible kinematic configurations for this kind of proton decay. In 
each configuration, all the Lorentz-violating effects are governed by a single parameter $\delta_{p}(\hat{p})-\delta_{w}(\hat{p})$. The first configuration isolates the $c$ and $d$ coefficients for a single decay product by having the other decay products be nonrelativistic; this makes it possible to obtain clean bounds on the coefficients for just that one species of particle. The second configuration is relevant if it is known that there is Lorentz violation in one sector only. This is an interesting case to consider theoretically, but the analysis does not lead to rigorous bounds on any physical coefficients. The third kinematic configuration applies when the proton does not actually decay, but rather emits a light neutral particle, as in vacuum Cerenkov radiation $p^{+} \rightarrow p^{+}+\gamma$, or $p^{+} \rightarrow p^{+}+\pi^{0}$.

In the first configuration, one of the daughter particles will carry away practically all of the proton's initial momentum $\vec{p}$, leaving the remaining one (or more) essentially at rest. This is generally not the way the momentum is divided up at the threshold for the reaction, but this configuration ensures that only the $\delta_{w}$ for a single species among the daughter particles will enter our calculations. The moving particles are essentially collinear, so only the $\delta_{w}(\hat{p})$ values for a single direction enter. Energy-momentum conservation requires that for a two-body decay at threshold,

$$
\left(1+\delta_{p}\right) p=\left(1+\delta_{1}\right) p+m_{2},
$$

where 1 and 2 label the daughter particles. We have dropped terms that are suppressed relative to the particle masses by powers of $m / p$. Moreover, because only one direction is involved, we have omitted the dependence of $\delta_{w}$ on $\hat{p}$.

Apparently, this process can occur at a momentum $p$ if $\delta_{p}-\delta_{1}=m_{2} / p$. A positive $\delta_{p}$ indicates that the initial proton with momentum $p$ has more energy than it would have in the Lorentz-invariant theory. If this momentum is transferred to a particle with a smaller $\delta_{1}$, there may be enough energy left over to create a particle of type 2 . If $\delta_{p}<\delta_{1}$, however, the process will never be allowed, so the observed absence of these kinds of decays can only give one-sided bounds on the various $\delta_{p}-\delta_{w}$. However, one-sided bounds on the $\delta_{w}(\hat{p})$ for different directions $\hat{p}$ can be used to place two-sided bounds on some of the individual $c^{w}$ and $d^{w}$ coefficients.

The generalization to more than two decay products is straightforward. If one particle still carries away all the momentum, the process can occur if $\delta_{p}-\delta_{1}>\left(\sum m_{w}\right) / p$, where $\sum m_{w}$ is the sum of the masses of all the other daughter particles. Usually this sum will be dominated by the mass of the heaviest product other than the one carrying away the momentum. It follows that the strength of the bounds we can place on the SME coefficients relevant to a particular species is determined by the masses of the other species that are produced along with it. The bounds for a particular species are better if the other particles involved are lighter.

Of particular interest is the three-body $\beta$-decay $p^{+} \rightarrow n+e^{+}+\nu_{e}$. The fact that protons of energy $E$ traveling in the direction $\hat{p}$ do not decay in this fashion places a bound

$$
\delta_{p}(\hat{p})-\delta_{n}(\hat{p})<\frac{m_{e}}{E}
$$


and this can be used to constrain some quantities in the neutron sector that have not previously been measured. For the most energetic observed cosmic rays, $m_{e} / E \lesssim 10^{-14}$. It is interesting that the constraint (5) is only $\mathcal{O}(\mathrm{m} / E)$, whereas most previous bounds on $\delta_{w}$ coming from high-energy observations were $\mathcal{O}\left(\mathrm{m}^{2} / E^{2}\right)$; this new energy dependence arises from the involvement of a nonrelativistic particle in the decay reaction.

The kinematic configuration in which all but one of the daughter particles is at rest is not generally the threshold configuration. However, it is a configuration in which the $\delta_{w}$ for only one decay products enters. If it is known that there is Lorentz violation for only one of the daughter particles, then the true threshold can be determined straightforwardly. While calculating this threshold, we shall assume that the particle with the Lorentzviolating dispersion relation is much heavier than the other particles produced alongside it, as is the neutron in $p^{+} \rightarrow n+e^{+}+\nu_{e}$.

In the threshold configuration, the heavy, Lorentz-violating particle again carries away most of the momentum, so it will be moving at an ultrarelativistic speed. However, since the other daughter particles are substantially less massive, they too can be highly relativistic, even though they carry only a small fraction of the total momentum. At threshold, the moving particles are again essentially collinear.

To lowest order, the lighter particles produced in the decay may actually be neglected. Neglecting them, the threshold at which the conversion of a proton into the heavy decay product becomes allowed is given approximately by

$$
\begin{aligned}
\sqrt{m_{p}^{2}+\left(1+2 \delta_{p}\right) p^{2}} & =\sqrt{m_{1}^{2}+\left(1+2 \delta_{1}\right) p^{2}} \\
\left(1+\delta_{p}\right) p+\frac{m_{p}^{2}}{2 p} & =\left(1+\delta_{1}\right) p+\frac{m_{1}^{2}}{2 p} .
\end{aligned}
$$

We have dropped terms that are suppressed beyond lowest order in $m / p$. The process can occur if $\delta_{p}-\delta_{1}=\left(m_{1}^{2}-m_{p}^{2}\right) / 2 p^{2}$, placing the threshold at approximately $p_{T}=$ $\sqrt{\left(m_{1}^{2}-m_{p}^{2}\right) / 2\left(\delta_{p}-\delta_{1}\right)}$.

If the other decay products are light enough, they will have little impact on the threshold value of $p$. If $m_{w} \ll \sqrt{m_{1}^{2}-m_{p}^{2}}$, then including an additional particle of species $w$ among the decay products only raises the threshold momentum by $\sqrt{2 /\left(\delta_{p}-\delta_{1}\right)} m_{w}$. The additional momentum is divided evenly between the heavy particle 1 and the light particle $w$. Each light daughter particle increases the threshold only slightly, with the net result that the threshold occurs at

$$
p_{T}=\sqrt{\frac{2}{\delta_{p}-\delta_{1}}}\left(\frac{\sqrt{m_{1}^{2}-m_{p}^{2}}}{2}+\sum m_{w}\right) .
$$

Unfortunately, this result is not very useful for placing bounds, because the analysis presupposes that Lorentz violation is only important for the heaviest decay product. If 
the values of $\delta_{w}$ for the other decay products are comparable to the $\delta_{1}$ corresponding to the heaviest product, the overall scale of the threshold is still $p_{T} \sim \sqrt{\left(m_{1}^{2}-m_{p}^{2}\right) /\left(\delta_{p}-\delta_{1}\right)}$. However, if Lorentz violation for the other particles is allowed to be larger, the threshold may increase dramatically. If the $\delta_{w}$ for the lighter particles grows large enough, the threshold configuration may become the first one considered, in which all but the heaviest particle are produced at rest.

If there were known to be no Lorentz violation in the electron or neutrino sectors, the analysis of the true threshold location would give a much tighter bound on $\delta_{p}-\delta_{n}$ than (5). The bounds on $\delta_{p}-\delta_{n}$ would be at the $10^{-22}$ level. However, Lorentz violation in the neutrino sector is very poorly constrained. Therefore, (5) represents the best bound that can be placed at the present time.

The third kinematic configuration we shall consider is one with a proton and a lighter neutral particle in the final state. Because there are only two species involved in the whole process, the threshold will again depend only on the combination $\delta_{p}-\delta_{1}$. When the particles are collinear, as they are at threshold, energy-momentum conservation dictates

$$
\left(1+\delta_{p}\right) p+\frac{m_{p}^{2}}{2 p}=\left(1+\delta_{p}\right)\left(p-p_{1}\right)+\frac{m_{p}^{2}}{2\left(p-p_{1}\right)}+\left(1+\delta_{1}\right) p_{1}+\frac{m_{1}^{2}}{2 p_{1}} .
$$

The threshold lies at the minimum of $p$ as a function of $p_{1}$; setting $d p / d p_{1}=0$ determines $\delta_{p}-\delta_{1}$ to be

$$
\delta_{p}-\delta_{1}=\frac{m_{p}^{2}}{2\left(p_{T}-p_{1}\right)^{2}}-\frac{m_{1}^{2}}{2 p_{1}^{2}} .
$$

With this $\delta_{p}-\delta_{1}$, and $m_{1} / m_{p} \ll 1$, energy-momentum conservation is satisfied for $p_{1}=$ $\left(2 m_{1}^{2} / m_{p}^{2}\right)^{1 / 3} p_{T} \ll p_{T}$. At threshold, the momentum carried away by the lighter particle is small compared with the momentum that remains with the proton.

If protons up to momentum $p$ do not radiate neutral particles in this way, we must therefore have $\delta_{p}-\delta_{1}<m_{p}^{2} / 2 p^{2}$. This bound has already been recognized for vacuum Cerenkov radiation [8], for which case $m_{1}=m_{\gamma}=0$. The Cerenkov threshold can also be calculated using the usual result that Cerenkov radiation is emitted when a charged particle moves faster than the phase speed of light in the same direction. We shall discuss the vacuum Cerenkov bounds in more detail shortly. However, the absence of the process $p^{+} \rightarrow p^{+}+\pi^{0}$ can also be used to place bounds on Lorentz violation in the pion sector, which are significantly better than any previous pion bounds. For heavier uncharged mesons, with masses comparable to $m_{p}$, the above analysis must be modified slightly, but the resulting bounds are at a similar level - worse only by an $\mathcal{O}(1)$ factor if there are no weak interactions involved in the process.

Having discussed the decay kinematics in several scenarios, we shall turn to determining the quantitative bounds on the $\delta$ parameters for various particles. Bounds on neutron parameters are of the greatest interest. However, there is one remaining issue, related to how long the various decay or emission processes we have discussed will take. Obviously, 
for bounds be based on a given reaction process, it must be possible for that reaction to occur in the travel time of a cosmic ray proton. However, this criterion is easily satisfied for any reaction that is mediated by either the strong or electromagnetic interaction. The rate of vacuum Cerenkov radiation was calculated in [14], and the process is extremely rapid. A strong process such as $p^{+} \rightarrow p^{+}+\pi^{0}$ will naturally occur even more quickly.

However, since the decay $p^{+} \rightarrow n+e^{+}+\nu_{e}$ is a weak interaction process, there might be a concern that it will not be rapid enough to occur during the primary proton's flight to Earth. Yet a proton traveling $10 \mathrm{Mpc}$ to Earth with an energy of $10^{11} \mathrm{GeV}$ experiences a proper time span greater than $10^{4} \mathrm{~s}$. This is much longer than the typical lifetime of weakly decaying hadrons, longer in particular than the neutron lifetime of approximately $880 \mathrm{~s}$.

In a Lorentz invariant theory, the rate at which a process occurs is determined by the matrix element, which depends on the details of the dynamics, and the phase space available to the outgoing particles. The rate is generally a rapidly increasing function of the available phase space. These statements continue to hold in theories with Lorentz violation [15]. The rate at which a proton decays is determined by the matrix element for the decay and the phase space available to the decay products in the initial proton's rest frame. In this frame, the Lorentz violation actually makes only a small correction to the phase space - provided that the energy available for the decay is known. The phase space $\Pi^{w}$ for a given decay product is equal to $\Pi_{0}^{w}\left[1+\mathcal{O}\left(\delta_{w}\right)\right]$, where $\Pi_{0}^{w}$ is the phase space for a Lorentz invariant particle with the same energy. For determining the approximate rate for a process, it is therefore a reasonable approximation to ignore the effects of Lorentz violation on the decay rate, except in the calculation of how much energy is released in the decay.

The amount of energy that is available for the daughter particles to carry away as kinetic energy is quite straightforward to calculate. To determine this available energy, we can (as in the second kinematical configuration considered above) begin by neglecting all particles produced in the decay except the heaviest one. In the laboratory frame, where the original proton is moving extremely rapidly, the heavy decay product's energy is $E_{w}=\left(1+\delta_{w}\right) p+\frac{m_{w}^{2}}{2 p}$. The energy of this configuration in the proton's rest frame is $E_{r}=\gamma_{p}\left(E_{w}-v_{p} p\right)$, where $v_{p}$ and $\gamma_{p}$ are the proton's velocity and Lorentz factor in the laboratory frame. To the required order, $v_{p}=\left(1+\delta_{p}\right)-\frac{m_{p}^{2}}{2 p^{2}}$ and $\gamma_{p}$ is simply $p / m_{p}$. In terms of the threshold momentum $p_{T}$ (which is the momentum at which $E_{r}=m_{p}$ ),

$$
E_{r}=\frac{m_{p}^{2}-m_{w}^{2}}{2 m_{p}} \frac{p^{2}}{p_{T}^{2}}+\frac{m_{p}^{2}+m_{w}^{2}}{2 m_{p}} .
$$

When this is less than the rest energy $m_{p}$ of the proton, the decay products will carry some kinetic energy in the center of mass frame. The amount of kinetic energy available 
to the decay products in this frame is

$$
m_{p}-E_{r}=\frac{m_{2}^{2}-m_{p}^{2}}{2 m_{p}}\left(\frac{p^{2}}{p_{T}^{2}}-1\right) \approx\left(m_{w}-m_{p}\right)\left(\frac{p^{2}}{p_{T}^{2}}-1\right),
$$

where the approximation in (12) is valid if $m_{w} \approx m_{p}$, which holds if the particle $w$ is a neutron. The inclusion of the other, lighter decay products decreases the available energy by a small amount.

If $p=2 p_{T}$, the available energy is greater than the neutron-proton mass difference of 1.29 MeV. Therefore, the phase space available for the decay at this energy is greater than the phase space for the decay of a stationary neutron. The matrix element for the proton $\beta$-decay process $p^{+} \rightarrow n+e^{+}+\nu_{e}$ near threshold is extremely similar to the usual matrix element for neutron $\beta$-decay. The matrix element is not suppressed by the smallness of the Lorentz violation. In fact, if the available energies for the processes are precisely the same (that is, if $m_{p}-E_{r}=m_{n}-m_{p}$ ), the only differences between the rates for the two reactions would come from small changes to the phase space and isospin-violating differences in the matrix elements. Since the available phase space and rate for the proton disintegration increase with $p$, above $p=2 p_{T}$, the proton will decay with a mean proper lifetime less than $880 \mathrm{~s}$. With a lifetime this short, nearly all the primary protons with more than twice the threshold energy will decay during their journeys. The weak nature of the decay is no impediment to the decay's ready occurrence during the time it takes for a cosmic ray proton to reach Earth.

We can therefore use (5) to place rigorous new bounds on Lorentz violation for neutrons. However, in order to establish new bounds, we must understand the existing bounds in both the neutron and proton sectors. We shall first consider the existing bounds based on vacuum Cerenkov radiation. If this process were allowed above some threshold, a proton with a higher energy would radiate away the excess energy extremely quickly [14]. If protons with energies up to $E$ are observed not to emit vacuum Cerenkov radiation, this translates into a bound on the quantity $\delta_{p}-\delta_{\gamma}$ that is $\mathcal{O}\left(m_{p}^{2} / E^{2}\right)$. The quantity $\delta_{\gamma}(\hat{p})$ is analogous to the fermionic $\delta(\hat{p})$, and it governs the phase speed of photons in the direction $\hat{p}$. $\delta_{\gamma}(\hat{p})$ depends on the $k_{F}$ coefficients in the Lorentz-violating Lagrange density for the electromagnetic sector,

$$
\mathcal{L}_{A}=-\frac{1}{4} F^{\mu \nu} F_{\mu \nu}-\frac{1}{4} k_{F}^{\mu \nu \rho \sigma} F_{\mu \nu} F_{\rho \sigma}
$$

However, the spin-dependent part of $\delta_{\gamma}$ has been shown to be extremely small using measurements of cosmological birefringence [16, 17, 18], and it may therefore be neglected. Moreover, for any one sector, the spin-independent part of $\delta$ may be defined away. We shall use this freedom to make the electromagnetic sector conventional. Our results can be adapted to a different convention with a nonzero $k_{F}$ simply by making the replacement $c^{\mu \nu} \rightarrow c^{\mu \nu}-\frac{1}{2} k_{F \alpha}{ }^{\mu \alpha \nu}$ in every matter sector. 
Existing cosmic ray data place bounds on the components of $\delta_{p}-\delta_{\gamma}$, which is just $\delta_{p}$ with our conventions. If protons of both helicities are part of the primary cosmic ray spectrum, these bounds apply to both helicities; if only one helicity is present, the bounds obviously apply only to that helicity. What is important is that any proton that is part of the primary cosmic ray flux is subject to these bounds.

Most of the details of the vacuum Cerenkov bounds are worked out in [8]. From the absence of vacuum Cerenkov radiation, there are two-sided bounds on the $-c_{(0 j)}^{p}+s d_{(0 j)}^{p}$ and $-c_{j k}^{p}+s d_{j k}^{p}$ coefficients appearing in $\delta_{p}$-if it is assumed that the isotropic term $-c_{00}^{p}+s d_{00}^{p}$ is small compared with the others. Two-sided bounds in the presence of a generic $c_{00}^{p}$ are impossible, because a large enough $c_{00}^{p}$ can render the proton MAV less than one for every direction and spin. The absence of vacuum Cerenkov radiation thus cannot constrain a positive $c_{00}^{p}$. However, if $-c_{00}^{p}+s d_{00}^{p} \approx 0$, the other coefficients are all separately bounded at the $2 \times 10^{-21}$ level. (If the anisotropic terms are assumed to vanish instead, there is a one-sided bound on the isotropic coefficient, $c_{00}^{p}-s d_{00}^{p}>-10^{-22}$.)

There are also bounds on $c^{p}$ and $d^{p}$ (as well as neutron coefficients) from laboratory experiments with atomic clocks - some of which are much stronger than the cosmic ray bounds, ranging from the $10^{-20}$ to $10^{-29}$ levels [19, 20, 21, 22, 23] -although most of the $d^{p}$ coefficients remain unconstrained. Yet laboratory experiments which rely on the Earth's rotation to search for anisotropic effects are typically insensitive to a number of SME coefficients. Moreover, the clock comparison experiments using hyperfine transitions have only been sensitive to forms of neutron boost invariance violations that also depend on the spin. The $c_{(T j)}^{n}$ coefficients, which characterize a spin-independent anisotropy in the way neutrons respond to Lorentz boosts, have never been studied.

If the isotropic part of the proton Lorentz violation is small, the preexisting bounds justify setting $\delta_{p}=0$ in (5), because the vacuum Cerenkov bounds in the proton sector are quite a bit tighter than the new bounds we shall be considering here. The remaining neutron bounds coming from (5), which have the form $-\delta_{n}(\hat{p})<m_{e} / E$, are based on precisely the same data set as the vacuum Cerenkov bounds on $\delta_{p}$. So the consequences for the individual neutron $c$ and $d$ coefficients are (unsurprisingly) similar. If there is no isotropic neutron term, each of the neutron coefficients $-c_{(0 j)}^{n}+s d_{(0 j)}^{n}$ or $-c_{j k}^{n}+s d_{j k}^{n}$ is bounded above and below by $5 \times 10^{-14}$. On the other hand, if there is exclusively isotropic neutron Lorentz violation (but still no isotropic proton Lorentz violation), $c_{00}^{n}-s d_{00}^{n}<$ $10^{-14}$. What is more, these bounds must hold for both helicities: $s= \pm 1$. This did not need to be the case for the proton bounds, since only one proton helicity had to be immune to vacuum Cerenkov radiation for some protons to reach Earth; however, if the proton $\beta$-decay process were allowed for even one daughter neutron helicity, the primary protons would all decay away. Invoking the bounds for both signs of $s$, we see that actually each $\left|c_{(0 j)}^{n}\right|+\left|d_{(0 j)}^{n}\right|$ or $\left|c_{j k}^{n}\right|+\left|d_{j k}^{n}\right|$ is bounded by $5 \times 10^{-14}$.

This gives us our primary result for the neutron sector. If the dominant forms of Lorentz violation are not isotropic, then the previously unmeasured neutron coefficients $\frac{1}{4}\left|c_{Q}^{n}\right|=\frac{1}{4}\left|\left(c_{X X}^{n}+c_{Y Y}^{n}-2 c_{Z Z}^{n}\right)\right|,\left|c_{(T X)}^{n}\right|,\left|c_{(T Y)}^{n}\right|,\left|c_{(T Z)}^{n}\right|, \frac{1}{2}\left|d_{(X Z)}^{n}\right|$, and $\left|d_{(T Z)}^{n}\right|$ are all bounded 
by $5 \times 10^{-14} . X, Y, Z$, and $T$ are the coordinates in the sun-centered celestial equatorial coordinate system in which bounds on Lorentz violation are conventionally expressed. If isotropic Lorentz violation is possible, then the optimal bounds are represented by the one-sided inequalities (5), with the $\hat{p}$ and $E$ values for each ultra-high-energy proton cosmic ray that has been observed. There is still sensitivity to (just not two-sided bounds on) the six neutron coefficients just mentioned.

Of course, the bounds are not actually limited to the neutron sector. Any baryon that can be produced via the $\beta$-decay of a proton is subject to similar bounds. This means baryons with charge 0 or +2 . There are bounds for non-baryonic charged particles as well, but they are weaker. The reason for this is that there must be a baryon among the daughter particles, and if this is not the particle of interest, its mass will dominate the $\sum m_{w}$ on which the strength of the bound depends. Because baryons are so much heavier than the electron, the bounds are worse by a factor of $2 \times 10^{3}$ for charge +1 particles (which can be created in reactions that have nucleons in the final states) or slightly more for charge -1 and -2 particles (which must be produced along with something heavier, like a $\Delta$ ). The resulting bounds on the $\delta_{w}$ parameters for various charged mesons and baryons are at the $10^{-10}$ level. This makes them slightly worse than bounds derived from certain other astrophysical observations for charged pions [24]; however, they are an improvement over previous bounds for almost all heavier species of hadrons [25]. (The $\Delta^{+}$is an exception; there are much better bounds on the MAV for this species because of its involvement with the GZK cutoff.)

Neutral particles that do not carry baryon number and can be produced singly by the strong interaction are a separate case. The absence of the process $p^{+} \rightarrow p^{+}+\pi^{0}$ gives strong new bounds on the Lorentz violation coefficients for neutral pions. The relevant Lagrangian for the pion field is

$$
\mathcal{L}_{\pi}=\frac{1}{2}\left(\partial^{\mu} \pi\right)\left(\partial_{\mu} \pi\right)+\frac{1}{2} k_{\mu \nu}^{\pi}\left(\partial^{\mu} \pi\right)\left(\partial^{\nu} \pi\right)-\frac{m^{2}}{2} \pi^{2} .
$$

The coefficients $k^{\pi}$ are analogous to the fermionic $c$, and $\delta_{\pi}(\hat{p})=-\frac{1}{2}\left[k_{00}^{\pi}+2 k_{j 0}^{\pi} \hat{p}_{j}+k_{j k}^{\pi} \hat{p}_{j} \hat{p}_{k}\right]$. Again assuming the isotropic component of the Lorentz violation is negligible, there are two-sided bounds on all the remaining $k^{\pi}$ coefficients, which must be smaller than $4 \times 10^{-21}$. These represent an eleven order of magnitude improvement over the neutral pion bounds from [24]. There should be similar (but slightly weaker) bounds for any heavier neutrals mesons that can be produced strongly. These include the pseudoscalar $\eta$ and $\eta^{\prime}$, as well as the vector $\rho^{0}, \omega$, and $\phi$. However, the full set of Lorentz-violating coefficients relevant for a massive, spin-1 particle has not been worked out.

If primary cosmic ray protons were undergoing $\beta$-decays, the positrons produced in the decay could also become part of the cosmic ray spectrum. The fact that there are observed to be very few electrons and positrons among the most energetic cosmic rays might be used to place bounds on the Lorentz violation coefficients for the electron sector. However, the resulting bounds would be at only the $10^{-10}$ level, significantly weaker than 
other astrophysical bounds on the same coefficients [26, 27], which constrain $c^{e}$ and $d^{e}$ at the $10^{-14}-10^{-17}$ levels.

This whole analysis presumes that it is known that the cosmic rays we observe are largely protons. However, the arguments that the spectrum is predominantly protonic are usually made in the context of a theory that is assumed to be Lorentz invariant. If primary cosmic ray protons above a certain energy may decay into neutrons, which are stable at that energy, the spectrum might change from proton-dominated to neutrondominated above the energy in question. Put another way, the decay $p^{+} \rightarrow n+e^{+}+\nu_{e}$ still has an energetic nucleon among the decay products; above the threshold for this decay, the neutron that is produced could simply replace the proton as part of the spectrum. While this is to some extent an accurate evaluation, it is important to realize that the proton decay would still leave a distinctive imprint on the observed cosmic ray spectrum. The reason is that the decay does not simply transfer all the primary proton's energy to the daughter neutron. The proton energy is split up between three particles, and so the neutrons produced via the decay will possess less energy than their protonic forbears. Moreover, if the initial proton is not too far above the threshold for the decay, the neutron's energy may even be below the threshold; in that case, the neutron itself will decay, back into an even lower energy proton. The net process in that case is $p^{+} \rightarrow p^{+}+e^{-}+e^{+}+\nu_{e}+\bar{\nu}_{e}$; the proton releases some of its energy in the form of lepton-antilepton pairs. In any case, if above some energy, the cosmic ray spectrum changed from protonic to neutronic, there would be a pronounced feature in the spectrum at that energy. Since there are no large unexplained features in the ultra-high-energy cosmic ray spectrum, we can safely rule out this possibility.

There is also the unlikely possibility that the highest energy cosmic rays are not protons, but rather are predominantly nuclei, most likely ${ }^{56} \mathrm{Fe}$. If this somehow turns out to be the case, the bounds on neutron Lorentz violation discussed here would be weakened somewhat. Proton to neutron conversion could still occur inside the nuclei if $\delta_{p}-\delta_{n}$ were large enough, but the resulting bounds would be less stringent, primarily because of the smaller momenta of the constituent hadrons. Each proton in an ${ }^{56} \mathrm{Fe}$ cosmic ray with energy $E$ carries a momentum of roughly $E / 56$. This decrease in momentum, in conjunction with the effects of nuclear binding, will probably lead to bounds that are worse by about two orders of magnitude. However, the ${ }^{56} \mathrm{Fe}$ scenario seems unlikely. Heavier primary cosmic rays will generally produce air showers with more muons, and this can be used to constrain the composition of the primary cosmic ray spectrum [28]. Estimates of the fraction of ${ }^{56} \mathrm{Fe}$ nuclei among the highest energy cosmic rays vary, but [29] placed an upper limit of $64 \%$ on the iron fraction. It is also possible that the cosmic rays might be produced initially as nuclei, which then undergo photodisintegration in flight. However, this would not significantly effect our bounds on Lorentz violation. If photodisintegration of ${ }^{56} \mathrm{Fe}$ is the source of primary cosmic ray protons, the protons will still propagate over megaparsec distances [30. Since the mean lifetime of protons with energies more than twice the decay threshold is significantly shorter than intergalactic 
travel times, the protons would still have time to decay in large numbers.

In summary, we have shown that the stability of primary cosmic ray protons with energies up the GZK cutoff has implications for Lorentz violation. That there were bounds on SME parameters coming from the protons' stability against vacuum Cerenkov radiation had already been observed. However, the fact that an energetic proton does not decay into a neutron places new bounds on Lorentz violation coefficients in the neutron sector, at the $m_{e} / E$ level. These bounds are most conveniently expressed in the absence of isotropic Lorentz violation, in which case six previously unmeasured neutron coefficients

are bounded by $5 \times 10^{-14}$; this includes the first constraints on spin-independent forms of boost anisotropy for neutrons. The absence of similar decay processes place comparable bounds on the coefficients for other charged baryons, while there are weaker bounds for charged hadrons that do not have baryon number $B=1$. For neutral mesons that can be produced via the strong interaction, the bounds are significantly better - at the $4 \times 10^{-21}$ level for neutral pions, for example. All this demonstrates the continued utility of highenergy astrophysical data for constraining Lorentz violation and possibly other forms of exotic physics.

\section{References}

[1] R. U. Abbasi, et al., Phys. Rev. Lett. 100, 101101 (2008).

[2] K. Greisen, Phys. Rev. Lett. 16, 748 (1966).

[3] G. T. Zatsepin, V. A. Kuz'min, JETP Lett. 4, 78 (1966) [Pis'ma Zh. Eksp. Teor. Fiz. 4, 114 (1966)].

[4] J. Abraham, et al., Science 318, 938 (2007).

[5] S. Coleman, S. L. Glashow, Phys. Rev. D 59, 116008 (1999).

[6] O. Gagnon, G. D. Moore, Phys. Rev. D 70, 065002 (2004).

[7] C. D. Carone, M. Sher, M. Vanderhaeghen, Phys. Rev. D 74, 077901 (2006).

[8] F. R. Klinkhamer, M. Risse, Phys. Rev. D 77, 016002 (2008); addendum Phys. Rev. D 77, 117901 (2008).

[9] D. Colladay, V. A. Kostelecký, Phys. Rev. D 55, 6760 (1997).

[10] D. Colladay, V. A. Kostelecký, Phys. Rev. D 58, 116002 (1998).

[11] Proceedings of the Fourth Meeting on CPT and Lorentz Symmetry, edited by V. A. Kostelecký (World Scientific, Singapore, 2008). 
[12] V. A. Kostelecký, N. Russell, arXiv:0801.0287.

[13] B. Altschul, D. Colladay, Phys. Rev. D 71, 125015 (2005).

[14] B. Altschul, Phys. Rev. Lett. 98, 041603 (2007).

[15] D. Colladay, V. A. Kostelecký, Phys. Lett. B 511209 (2001).

[16] V. A. Kostelecký, M. Mewes, Phys. Rev. Lett. 87, 251304 (2001).

[17] V. A. Kostelecký, M. Mewes, Phys. Rev. Lett. 97, 140401 (2006).

[18] V. A. Kostelecký, M. Mewes, Phys. Rev. Lett. 99, 011601 (2007).

[19] V. A. Kostelecký, C. D. Lane, Phys. Rev. D 60, 116010 (1999).

[20] D. Bear, R. E. Stoner, R. L. Walsworth, V. A. Kostelecký, C. D. Lane, Phys. Rev. Lett. 85, 5038 (2000).

[21] F. Canè, D. Bear, D. F. Phillips, M. S. Rosen, C. L. Smallwood, R. E. Stoner, R. L. Walsworth, V. A. Kostelecký, Phys. Rev. Lett. 93, 230801 (2004).

[22] P. Wolf, F. Chapelet, S. Bize, A. Clairon, Phys. Rev. Lett. 96, 060801 (2006).

[23] T. W. Kornack, G. Vasilakis, M. V. Romalis, in [11].

[24] B. Altschul, Phys. Rev. D 77, 105018 (2008).

[25] B. Altschul, Astropart. Phys. 28, 380 (2007).

[26] B. Altschul, Phys. Rev. Lett. 96, 201101 (2006).

[27] B. Altschul, Phys. Rev. D 75, 041301 (R) (2007).

[28] M. Risse, P. Homola, R. Engel, D. Gora, D. Heck, J. Pekala, B. Wilczyńska, H. Wilczyński, Phys. Rev. Lett. 95, 171102 (2005).

[29] M. T. Dova, A. A. Watson, A. G. Mariazzi, astro-ph/0512408.

[30] L. A. Anchordoqui, M. T. Dova, L. N. Epele, J. D. Swain, Phys. Rev. D 57, 7103 (1998). 